\title{
On Toeplitz operators with quasihomogeneous symbols
}

\author{
By
}

ISSAM LOUHICHI and LOVA ZAKARIASY

\begin{abstract}
In this paper, we give some basic results concerning Toeplitz operators whose symbol is of the form $e^{i p \theta} \phi$, where $\phi$ is a radial function, then use these results to characterize all Toeplitz operators which commute with them.
\end{abstract}

1. Introduction. Let $\mathbb{D}$ denote the open unit disc in the complex plane and let $d A(z)=$ $\frac{1}{\pi} r d r d \theta$ be normalized Lebesgue area measure on $\mathbb{D}$. The Bergman space $L_{a}^{2}$ is the subset of $L^{2}(\mathbb{D}, d A)$, consisting of analytic functions on $\mathbb{D}$. Let $P$ be the operator of orthogonal projection from $L^{2}(\mathbb{D}, d A)$ onto $L_{a}^{2}$. For a function $\varphi \in L^{\infty}(\mathbb{D}, d A)$, we define the Toeplitz operator $T_{\varphi}: L_{a}^{2} \mapsto L_{a}^{2}$ with symbol $\varphi$ by

$$
T_{\varphi}(f)=P(\varphi f) \text {. }
$$

It is well known that, if $K_{z}(w)=(1-\bar{z} w)^{-2}$ is the Bergman reproducing kernel, then

$$
T_{\varphi}(f)(z)=\int_{\mathbb{D}} \varphi(w) f(w) \overline{K_{z}(w)} d A(w), \quad z \in \mathbb{D} .
$$

In 1964, Brown and Halmos [3] showed that on the Hardy space, two bounded Toeplitz operators $T_{\varphi}$ and $T_{\psi}$ commute if and only if: (i) both $\varphi$ and $\psi$ are analytic, or (ii) both $\bar{\varphi}$ and $\bar{\psi}$ are analytic, or (iii) one is a linear function of the other. In [1] Axler and Ččković proved that if the two symbols are bounded harmonic functions, then the same result is also true for Toeplitz operators on the Bergman space. Recently, with Rao [2], they proved that if $\varphi$ is a bounded analytic function and if there exists a bounded function $\psi$ such that $T_{\varphi}$ and $T_{\psi}$ commute on $L_{a}^{2}$, then $\psi$ must be analytic too. In [8] and [9] Vasilevski gave the description of many (geometrically defined) classes of commuting Toeplitz operators.

The situation with a general symbol is rather more complicated. Let $\varphi, \psi \in L^{1}(\mathbb{D}, d A)$ be radial functions, i.e. $\varphi(z)=\varphi(|z|), z \in \mathbb{D}$. It is well known and easy to see that two

Mathematics Subject Classification (1991): Primary 47B35; Secondary 47L80. 
Toeplitz operators with radial symbols commute. We show here that if $p$ and $s$ are integers such that $p s \leqq 0$, then the Toeplitz operators with symbols $e^{i p \theta} \varphi$ and $e^{i s \theta} \psi$ commute only in certain trivial cases. The case $p s \geqq 0$ is treated for $\varphi=r^{m}$ in [4]. We note that the part (b) of Theorem 3 in [4] is not correct as stated.

2. Some basic results about quasihomogeneous symbols. An operator that will arise in our study of Toeplitz operators is the Mellin transform, defined for any function $\varphi \in$ $L^{1}([0,1] ; r d r)$, by the formula

$$
\widehat{\varphi}(z)=\int_{0}^{1} \varphi(r) r^{z-1} d r,
$$

which is a bounded holomorphic function in the half plane $\{z: \operatorname{Re} z>2\}$. It is known that if there exists a sequence $\left(n_{k}\right)_{k \geqq 0} \subset \mathbb{N}$, such that

$$
\widehat{\varphi}\left(n_{k}\right)=0 \text { and } \sum_{k \geqq 0} \frac{1}{n_{k}}=\infty
$$

then by the Muntz-Szasz theorem (see [7]), $\varphi=0$.

A function $f$ is said to be quasihomogeneous of degree $k$ if and only if

$$
f\left(r e^{i \theta}\right)=e^{i k \theta} \varphi(r),
$$

where $\varphi$ is a radial function (see [6]). A direct calculation gives the following lemma which we shall use often.

Lemma 1. Let $p \geqq 0$ an integer and $\varphi$ a bounded radial function. Then, for all $n=0,1,2, \ldots$ :

$$
\begin{aligned}
T_{e^{i p \theta} \varphi}\left(z^{n}\right) & =2(n+p+1) \widehat{\varphi}(2 n+p+2) z^{n+p} ; \\
T_{e^{-i p \theta} \varphi}\left(z^{n}\right) & = \begin{cases}0 & \text { if } 0 \leqq n \leqq p-1 \\
2(n-p+1) \widehat{\varphi}(2 n-p+2) z^{n-p} & \text { if } n \geqq p .\end{cases}
\end{aligned}
$$

Next, we introduce the notion of radialization (see [10]). If $f \in L^{1}(\mathbb{D}, d A$ ), we define the "radialization" of $f$ by

$$
\operatorname{rad}(f)(z)=\frac{1}{2 \pi} \int_{0}^{2 \pi} f\left(e^{i t} z\right) d t .
$$

It is easy to see that a function $f$ is radial if and only if $\operatorname{rad}(f)=f$. 
Lemma 2. For a bounded function $f$ and for all integers $m, n \geqq 0$ :

$$
\left\langle T_{\operatorname{rad}(f)} z^{n}, z^{m}\right\rangle= \begin{cases}\left\langle T_{f} z^{m}, z^{m}\right\rangle & \text { if } n=m \\ & \text { if } n \neq m .\end{cases}
$$

Pr o of. Writing out the integrals, changing the order of integration and making a change of variable, we have for all integers $m, n \geqq 0$,

$$
\begin{aligned}
\left\langle T_{\operatorname{rad}(f)} z^{n}, z^{m}\right\rangle & =\frac{1}{2 \pi} \int_{0}^{2 \pi} \int_{\mathbb{D}} f\left(e^{i t} z\right) z^{n} \bar{z}^{m} d A(z) d t \\
& =\frac{1}{2 \pi}\left(\int_{0}^{2 \pi} e^{i(m-n) t} d t\right) \int_{\mathbb{D}} f(w) w^{n} \bar{w}^{m} d A(w) \\
& =\frac{1}{2 \pi}\left(\int_{0}^{2 \pi} e^{i(m-n) t} d t\right)\left\langle T_{f} z^{n}, z^{m}\right\rangle \\
& = \begin{cases}\left\langle T_{f} z^{m}, z^{m}\right\rangle & \text { if } n=m \\
0 & \text { if } n \neq m . \quad \square\end{cases}
\end{aligned}
$$

Proposition 3. A bounded function $f$ is quasihomogeneous of degree $p \in \mathbb{Z}$ if and only if, for all integers $n \geqq 0$, there exists $\lambda_{n} \in \mathbb{C}$ such that

$$
T_{f}\left(z^{n}\right)= \begin{cases}0 & \text { if } n<\max (-p, 0) \\ \lambda_{n} z^{n+p} & \text { if } n \geqq \max (-p, 0) .\end{cases}
$$

Proof. Let $f$ be a quasihomogeneous function of degree $p$, i.e., $f=e^{i p \theta} \varphi$, where $\varphi$ is a radial function. The necessity of condition (1) is a direct consequence of Lemma 1.

Conversely, suppose that equation (1) is true for any $p \in \mathbb{Z}$. Then, for any positive integers $n$ and $m$ :

if $p \geqq 0$

$$
\left\langle T_{\bar{z} p} f z^{n}, z^{m}\right\rangle=\left\langle f z^{n}, z^{m+p}\right\rangle= \begin{cases}0 & \text { if } n \neq m \\ \frac{\lambda_{m}}{m+p+1} & \text { if } n=m\end{cases}
$$

and if $p<0$

$$
\left\langle T_{z^{-p} f} z^{n}, z^{m}\right\rangle=\left\langle f z^{n-p}, z^{m}\right\rangle= \begin{cases}0 & \text { if } n \neq m \\ \frac{\lambda_{m-p}}{m+1} & \text { if } n=m\end{cases}
$$


Thus, if we note

$$
\varphi=\left\{\begin{array}{ll}
\bar{z}^{p} f & \text { if } p \geqq 0 \\
z^{-p} f & \text { if } p<0
\end{array},\right.
$$

then by Lemma 2 , we have for all integers $m, n \geqq 0$,

$$
\left\langle T_{\operatorname{rad}(\varphi)} z^{n}, z^{m}\right\rangle=\left\langle T_{\varphi} z^{n}, z^{m}\right\rangle
$$

hence, $T_{\operatorname{rad}(\varphi)}=T_{\varphi}$. Thus $\operatorname{rad}(\varphi)=\varphi$ and $\varphi$ is a radial function. But this easily implies that $f$ is a quasihomogeneous function of degree $p$.

Proposition 4. Let $f_{1}$ and $f_{2}$ be two quasihomogeneous bounded functions of degrees $p$ and $s$ respectively. If there exists a function $h$ such that $T_{f_{1}} T_{f_{2}}=T_{h}$, then $h$ is $a$ quasihomogeneous function of degree $p+s$.

Proof. Let $\varphi_{1}$ and $\varphi_{2}$ be the two radial functions, such that

$$
f_{1}=e^{i p \theta} \varphi_{1} \quad \text { and } \quad f_{2}=e^{i s \theta} \varphi_{2} .
$$

By Lemma 1, if $p$ and $s$ are greater than or equal to 0 , then for all $n \geqq 0$, there exists $\lambda_{n} \in \mathbb{C}$ such that

$$
T_{e^{i p \theta} \varphi_{1}} T_{e^{i s \theta} \varphi_{2}}\left(z^{n}\right)=\lambda_{n} z^{n+p+s} .
$$

When $p \geqq 0$ and $s<0$, we have

$$
T_{e^{i p \theta} \varphi_{1}} T_{e^{i s \theta} \varphi_{2}}\left(z^{n}\right)= \begin{cases}0 & \text { if } n<-s \\ \lambda_{n} z^{n+p+s} & \text { if } n \geqq-s .\end{cases}
$$

So, if $T_{f_{1}} T_{f_{2}}=T_{h}$ then by Proposition $3, h$ is a quasihomogeneous function of degree $p+s$.

If $p$ and $s$ are both negative, or if $p<0$ and $s \geqq 0$, then by considering the adjoint operator we obtain the same result.

Proposition 4 helps us to identify the Toeplitz operators which are idempotents. The following corollary is an immediate consequence.

Corollary 5. If $f$ is a quasihomogeneous function of degree different from 0 and if $T_{f}^{2}=T_{f}$, then $f=0$.

3. Commuting Toeplitz operators. In this section, we will see that two Toeplitz operators with quasihomogeneous symbols such that the signs of their quasihomogeneous degrees are opposite commute only in the trivial case, i.e., if one of them is the constant operator. Then Remark 12 shows that a function in $L^{2}(\mathbb{D}, d A)$ whose polar decomposition has only negative components cannot commute with a non trivial Toeplitz operator whose symbol is of positive quasihomogeneous degree. 
Proposition 6. Let $\psi$ be a bounded radial function and $e^{i p \theta} \phi$ a quasihomogeneous bounded function of degree $p>0$. If

$$
T_{\psi} T_{e^{i p \theta} \phi}=T_{e^{i p \theta} \phi} T_{\psi}
$$

then, $\phi=0$ or $\psi$ is a constant.

Proof. If $T_{e^{i p \theta} \phi}$ and $T_{\psi}$ commute, then for all $n \geqq 0$,

$$
\begin{gathered}
(2 n+2 p+2) \widehat{\phi}(2 n+p+2) \widehat{\psi}(2 n+2 p+2) \\
=(2 n+2) \widehat{\phi}(2 n+p+2) \widehat{\psi}(2 n+2) .
\end{gathered}
$$

Let $E=\{n: \widehat{\phi}(2 n+p+2)=0\}$. If $\sum_{n \in E} \frac{1}{n}=\infty$, then $\phi=0$, as discussed in Section 2 .

Otherwise, $\sum_{n \in E^{c}} \frac{1}{n}=\infty$, where $E^{c}$ is the complement of $E$ in $\mathbb{N}$, and so we have

$$
(2 n+2 p+2) \widehat{\psi}(2 n+2 p+2)=(2 n+2) \widehat{\psi}(2 n+2), \forall n \in E^{c} .
$$

This implies that

$$
(z+2 p) \widehat{\psi}(z+2 p)=z \widehat{\psi}(z), \forall z \in\{\operatorname{Re} z>0\} .
$$

For any integer $n_{0}$ greater than 0 , the last equation gives us

$$
\left(n_{0}+2 k p\right) \widehat{\psi}\left(n_{0}+2 k p\right)=n_{0} \widehat{\psi}\left(n_{0}\right), \quad \forall k \in \mathbb{N} .
$$

If we denote by $C$ the constant $n_{0} \widehat{\psi}\left(n_{0}\right)$, we obtain

$$
\widehat{\psi}\left(n_{0}+2 k p\right)=\frac{C}{n_{0}+2 k p}=C \widehat{\mathbb{1}}\left(n_{0}+2 k p\right), \quad \forall k \in \mathbb{N}
$$

and so, $\psi$ is equal to $C 11$.

R e m a r k 7. If $p<0$ the same result is true by considering the adjoint of the operator.

Proposition 8. Let $p \geqq s$ be two integers greater than 0 and $\phi$ and $\psi$ two bounded radial functions. If

$$
T_{e^{i p \theta} \phi} T_{e^{-i s \theta} \psi}=T_{e^{-i s \theta} \psi} T_{e^{i p \theta} \phi}
$$

then, $\phi=0$ or $\psi=0$.

Proof. If $T_{e^{i p \theta} \phi}$ and $T_{e^{-i s \theta} \psi}$ commute, then, for each $n \geqq 0$,

(a) $\widehat{\phi}(2 n+p+2) \widehat{\psi}(2 n+2 p-s+2)=0, \quad$ if $n \leqq s-1$

(b) $\widehat{\phi}(2 n+p+2) \widehat{\psi}(2 n+2 p-s+2)=C_{n} \widehat{\phi}(2 n+p-2 s+2) \widehat{\psi}(2 n-s+2)$ if $n \geqq s$.

where $C_{n}=\frac{n-s+1}{n+p+1}$. 
Now, equation (a) implies that for all $n_{0} \leqq s-1$, there exists a sequence $\left(n_{k}\right)_{k \in \mathbb{N}}$, which is defined by $n_{k+1}=n_{k}+s$ or $n_{k}+p$, such that

$$
\widehat{\phi}\left(2 n_{k}+p+2\right) \widehat{\psi}\left(2 n_{k}+2 p-s+2\right)=0 .
$$

It is clear that

$$
\sum_{k \in \mathbb{N}} \frac{1}{2 n_{k}+1}=\infty
$$

Let $E_{1}=\left\{k: \widehat{\phi}\left(2 n_{k}+p+2\right)=0\right\}$ and $E_{2}=\left\{k: \widehat{\psi}\left(2 n_{k}+2 p-s+2\right)=0\right\}$. Since

$$
\sum_{k \in \mathbb{N}} \frac{1}{2 n_{k}+1} \leqq \sum_{k \in E_{1}} \frac{1}{2 n_{k}+1}+\sum_{k \in E_{2}} \frac{1}{2 n_{k}+1}
$$
we see that at least one of the series $\sum_{k \in E_{1}} \frac{1}{2 n_{k}+1}$ and $\sum_{k \in E_{2}} \frac{1}{2 n_{k}+1}$ diverges, and so $\phi=0$
or $\psi=0 . \quad \square$

This result is not true if both of the integers $p$ and $s$ are positive. There are lots of examples of functions of positive quasihomogeneous degree which are the symbols of commuting Toeplitz operators (see [4]). In the general case we have the following proposition.

Proposition 9. Let $p, s$ be two integers greater than 0 and $\phi \neq 0$ a bounded radial function. If there exists a bounded radial function $\psi$ not identically zero, such that

$$
T_{e^{i p \theta} \phi} T_{e^{i s \theta} \psi}=T_{e^{i s \theta} \psi} T_{e^{i p \theta} \phi}
$$

then, $\psi$ is unique up to a constant factor.

Proof. Suppose there exist two functions $\psi_{1} \neq 0$ and $\psi_{2} \neq 0$, such that

$$
\begin{aligned}
& T_{e^{i p \theta} \phi} T_{e^{i s \theta} \psi_{1}}=T_{e^{i s \theta} \psi_{1}} T_{e^{i p \theta} \phi}, \\
& T_{e^{i p \theta} \phi} T_{e^{i s \theta} \psi_{2}}=T_{e^{i s \theta} \psi_{2}} T_{e^{i p \theta} \phi} .
\end{aligned}
$$

This is equivalent to:

$$
\left\{\begin{array}{l}
\widehat{\phi}(2 n+p+2 s+2) \widehat{\psi_{1}}(2 n+s+2) \\
=C_{n} \widehat{\phi}(2 n+p+2) \widehat{\psi_{1}}(2 n+2 p+s+2) \\
\widehat{\phi}(2 n+p+2 s+2) \widehat{\psi_{2}}(2 n+s+2) \\
=C_{n} \widehat{\phi}(2 n+p+2) \widehat{\psi_{2}}(2 n+2 p+s+2)
\end{array}\right.
$$

with $C_{n}=\frac{n+p+1}{n+s+1}$. From these equalities, one obtains:

$$
\widehat{r^{s} \psi_{1}}(2 n+2) \widehat{r^{s} \psi_{2}}(2 n+2 p+2)=\widehat{r^{s} \psi_{1}}(2 n+2 p+2) \widehat{r^{s} \psi_{2}}(2 n+2)
$$

for all $n$ in the set $E=\{n \in \mathbb{N}: \widehat{\phi}(2 n+2 s+p+2) \widehat{\phi}(2 n+p+2) \neq 0\}$. 
Clearly, we have $\sum_{n \in E} \frac{1}{n}=\infty$ because we supposed that $\phi \neq 0$.

From this and equation (3), we have

$$
\widehat{r^{s} \psi_{1}}(z) \widehat{r^{s} \psi_{2}}(z+2 p)=\widehat{r^{s} \psi_{1}}(z+2 p) \widehat{r^{s} \psi_{2}}(z), \forall z \in\{\operatorname{Re} z>-s\} .
$$

Now, since $\psi_{1}$ is not identically zero, there exists an integer $n_{0}$ such that

$$
\widehat{r^{s} \psi_{1}}\left(n_{0}\right) \neq 0 \text {. }
$$

Let $F=\left\{k \in \mathbb{N}: \widehat{r^{s} \psi_{1}}\left(n_{0}+2 k p\right)=0\right\}$. If $\sum_{k \in F} \frac{1}{n_{0}+2 k p}=\infty$, then equation (4) implies that $\widehat{r^{s} \psi_{2}}\left(n_{0}+2 k p\right)=0$ for all $k \in F$. Thus, $r^{s} \psi_{2}=0$ and so $\psi_{2}=0$. Otherwise, we have $\sum_{k \in F^{c}} \frac{1}{n_{0}+2 k p}=\infty$ and so by equation (4) we obtain that

$$
\left(\widehat{r^{s} \psi_{2}}-\lambda \widehat{r^{s} \psi_{1}}\right)\left(n_{0}+2 k p\right)=0, \quad \text { where } \lambda=\frac{\widehat{r^{s} \psi_{2}}\left(n_{0}\right)}{\widehat{r^{s} \psi_{1}}\left(n_{0}\right)} .
$$

Thus, in this case, $\psi_{2}=\lambda \psi_{1}$.

Now, let $\mathcal{R}$ be the space of functions which are square integrable in $[0,1]$ with respect to the measure $r d r$. By using the fact that the trigonometric polynomials are dense in $L^{2}(\mathbb{D}, d A)$ and that for $k_{1} \neq k_{2}, e^{i k_{1} \theta} \mathcal{R}$ is orthogonal to $e^{i k_{2} \theta} \mathcal{R}$, one see that:

$$
L^{2}(\mathbb{D}, d A)=\bigoplus_{k \in \mathbb{Z}} e^{i k \theta} \mathcal{R}
$$

Thus, each function $\psi \in L^{2}(\mathbb{D}, d A)$ can be written as (see [4]):

$$
\psi\left(r e^{i \theta}\right)=\sum_{k \in \mathbb{Z}} e^{i k \theta} \psi_{k}(r), \quad \text { where } \psi_{k} \in \mathcal{R} .
$$

Moreover, if $\psi \in L^{\infty}(\mathbb{D}, d A) \subset L^{2}(\mathbb{D}, d A)$ then for each $r \in[0,1)$,

$$
\left|\psi_{k}(r)\right|=\left|\int_{0}^{2 \pi} \psi\left(r e^{i \theta}\right) e^{-i k \theta} \frac{d \theta}{2 \pi}\right| \leqq \sup _{z \in \mathbb{D}}|\psi(z)|, \quad \forall k \in \mathbb{Z}
$$

hence, the functions $\psi_{k}$ are bounded in the disk.

Lemma 10. Let $e^{i p \theta} \phi$ be a bounded function of quasihomogeneous degree $p \geqq 0$ and let

$$
\psi\left(r e^{i \theta}\right)=\sum_{k \in \mathbb{Z}} e^{i k \theta} \psi_{k}(r) \in L^{\infty}(\mathbb{D}, d A)
$$

Then,

$$
T_{\psi} T_{e^{i p \theta} \phi}=T_{e^{i p \theta} \phi} T_{\psi} \Longleftrightarrow T_{e^{i k \theta} \psi_{k}} T_{e^{i p \theta} \phi}=T_{e^{i p \theta} \phi} T_{e^{i k \theta} \psi_{k}}, \quad \forall k \in \mathbb{Z}
$$


Proof. Since

$$
T_{\psi} T_{e^{i p \theta} \phi}\left(z^{n}\right)=\sum_{k+p+n \geqq 0} T_{e^{i k \theta} \psi_{k}} T_{e^{i p \theta} \phi} z^{n}
$$

and

$$
T_{e^{i p \theta} \phi} T_{\psi}\left(z^{n}\right)=\sum_{k+n \geqq 0} T_{e^{i p \theta} \phi} T_{e^{i k \theta} \psi_{k}} z^{n}
$$

then, for each couple $(m, n) \in \mathbb{N}^{2}$ :

$$
\left\langle T_{\psi} T_{e^{i p \theta} \phi} z^{n}, z^{m}\right\rangle=\left\langle T_{e^{i(m-n-p) \theta}} \psi_{m-n-p} T_{e^{i p \theta} \phi} z^{n}, z^{m}\right\rangle
$$

and

$$
\left\langle T_{e^{i p \theta} \phi} T_{\psi} z^{n}, z^{m}\right\rangle=\left\langle T_{e^{i p \theta} \phi} T_{e^{i(m-n-p) \theta} \psi_{m-n-p}} z^{n}, z^{m}\right\rangle .
$$

On the other hand,

$$
\begin{aligned}
& \left\langle T_{e^{i p \theta} \phi} T_{e^{i k \theta} \psi_{k}} z^{n}, z^{m}\right\rangle \\
& = \begin{cases}0 & \text { if } k \neq m-n-p \\
\left\langle T_{e^{i p \theta} \phi} T_{e^{i(m-n-p) \theta} \psi_{m-n-p}} z^{n}, z^{m}\right\rangle & \text { if } k=m-n-p\end{cases} \\
& = \begin{cases}0 & \text { if } k \neq m-n-p \\
\left\langle T_{e^{i p \theta} \phi} T_{\psi} z^{n}, z^{m}\right\rangle & \text { if } k=m-n-p\end{cases}
\end{aligned}
$$

and

$$
\begin{aligned}
& \left\langle T_{e^{i k \theta} \psi_{k}} T_{\left.e^{i p \theta} \phi z^{n}, z^{m}\right\rangle}\right. \\
& = \begin{cases}0 & \text { if } k \neq m-n-p \\
\left\langle T_{e^{i(m-n-p) \theta} \psi_{m-n-p}} T_{\left.e^{i p \theta}{ } z^{n}, z^{m}\right\rangle}\right. & \text { if } k=m-n-p\end{cases} \\
& =\left\{\begin{array}{ll}
0 & \text { if } k \neq m-n-p \\
\left\langle T_{\psi} T_{\left.e^{i p \theta} \phi z^{n}, z^{m}\right\rangle}\right. & \text { if } k=m-n-p
\end{array} .\right.
\end{aligned}
$$

This shows that, if $T_{e^{i p \theta} \phi} T_{\psi}=T_{\psi} T_{e^{i p \theta} \phi}$, then for each $(m, n) \in \mathbb{N}^{2}$ and for all $k \in \mathbb{Z}$,

$$
\left\langle T_{e^{i p \theta} \phi} T_{e^{i k \theta} \psi_{k}} z^{n}, z^{m}\right\rangle=\left\langle T_{e^{i k \theta} \psi_{k}} T_{e^{i p \theta} \phi} z^{n}, z^{m}\right\rangle,
$$

and so $T_{e^{i p \theta} \phi} T_{e^{i k \theta} \psi_{k}}=T_{e^{i k \theta} \psi_{k}} T_{e^{i p \theta} \phi}, \forall k \in \mathbb{Z}$. 
Conversely, if for all $k \in \mathbb{Z}, T_{e^{i p \theta} \phi} T_{e^{i k \theta} \psi_{k}}=T_{e^{i k \theta} \psi_{k}} T_{e^{i p \theta} \phi}$, then in particular, for any pair $m, n \in \mathbb{N}$, if $k=m-n-p$, we have:

$$
\left\langle T_{e^{i p \theta} \phi} T_{e^{i(m-n-p) \theta} \psi_{m-n-p}} z^{n}, z^{m}\right\rangle=\left\langle T_{e^{i(m-n-p) \theta} \psi_{m-n-p}} T_{e^{i p \theta} \phi} z^{n}, z^{m}\right\rangle .
$$

Thus, by formulas (6) and (7),

$$
\left\langle T_{e^{i p \theta} \phi} T_{\psi} z^{n}, z^{m}\right\rangle=\left\langle T_{\psi} T_{e^{i p \theta} \phi} z^{n}, z^{m}\right\rangle
$$

for all $m$ and $n$ in $\mathbb{N}$. Finally, $T_{e^{i p \theta} \phi} T_{\psi}=T_{\psi} T_{e^{i p \theta} \phi}$.

Theorem 11. Let $e^{i p \theta} \phi$ be a non-zero bounded function of quasihomogeneous degree $p>0$. If

$$
\psi\left(r e^{i \theta}\right)=\sum_{k \in \mathbb{Z}} e^{i k \theta} \psi_{k}(r) \in L^{\infty}(\mathbb{D}, d A)
$$

is such that $T_{\psi} T_{e^{i p \theta} \phi}=T_{e^{i p \theta} \phi} T_{\psi}$, then $\psi_{k}=0$ for all $k<0$.

Proof. By Lemma 10, $T_{\psi} T_{e^{i p \theta} \phi}=T_{e^{i p \theta} \phi} T_{\psi}$ implies that $T_{e^{i k \theta} \psi_{k}}$ commutes with $T_{e^{i p \theta} \phi}$ for each $k \in \mathbb{Z}$. Thus, if $k<0$ then the Proposition 8 gives $\psi_{k}=0$ for all $k<0$.

Remark 12. Let $\psi$ be as in Theorem 11 and $\phi$ a non constant bounded radial function such that $T_{\psi} T_{\phi}=T_{\phi} T_{\psi}$. Then, Lemma 10 gives us $T_{\phi} T_{e} i k \theta \psi_{\psi_{k}}=T_{e^{i k \theta} \psi_{k}} T_{\phi}$ for all $k \in \mathbb{Z}$. Now, Proposition 6 implies that $\psi_{k}=0$ for all $k>0$, while Remark 7 shows that $\psi_{k}=0$ for all $k<0$. So $\psi=\psi_{0}$.

This remark gives another proof of Theorem 6 in [4].

Acknowledgement. The authors would like to thank Elizabeth Strouse for the useful discussions with her.

\section{References}

[1] S. AXLER and Z. CUCKOviC, Commuting Toeplitz operators with harmonic symbols. Integral Equation Operator Theory 14, 1-12 (1991).

[2] S. AXLER, Z. CUCKOVIC and N. V. RAO, Commutants of analytic Toeplitz operators on the Bergman space. Proc. Amer. Math. Soc. 128, 1951-1953 (2000).

[3] A. BRown and P. R. Halmos, Algebraic properties of Toeplitz operators. J. Reine Angew. Math. 213, 89-102 (1963).

[4] Z. CUCKOVIC and N. V. RAO, Mellin transform, monomial symbols and commuting Toeplitz operators. J. Funct. Anal. 154(1), 195-214 (1998).

[5] S. GRUDSKY and N. VASILEVSKI, Bergman-Toeplitz operators: Radial component influence. Integral Equation Operator Theory 40, 16-33 (2001).

[6] I. LouHICHI, E. StrousE and L. ZAKARIASY, Products of Toeplitz operators on the Bergman space. Preprint.

[7] W. Rudin, Real and Complex analysis. Third edition, New York 1987.

[8] N. VASILEVSKI, Toeplitz Operator on the Bergman Spaces: Inside-the-Dommain Effects. Contemp. Math. 289, 79-146 (2001). 
[9] N. VASILEVSKI, Bergman space structure, commutative algebras of Toeplitz operators and hyperbolic geometry. Integral Equation Operator Theory 46, 235-251 (2003).

[10] N. ZorboskA, The Berezin transform and radial operators. Proc. Amer. Math. Soc. 131, 793-800 (2003).

Received: 12 June 2004; revised: 27 January 2005

I. Louhichi

UFR de Mathématiques Informatiques

Université Bordeaux I

351 cours de la libération

F-33405 Talence Cedex

France

louhichi@math.u-bordeaux1.fr
L. Zakariasy

High Institute of Technology

201 Diego-Suarez

Madagascar

zakarias@ refer.mg 